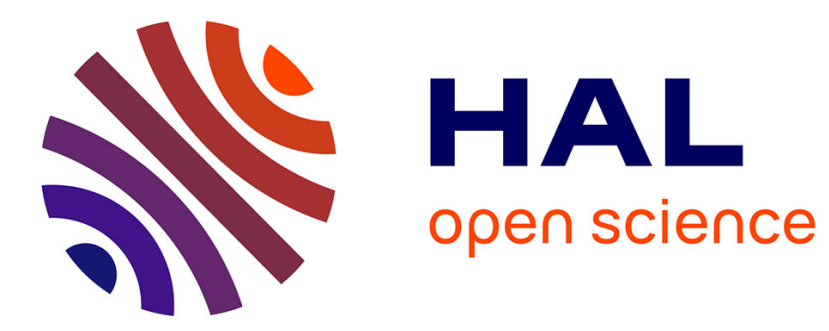

\title{
Self-preservation in stratified momentum wakes
}

Patrice Meunier, Peter Diamessis, Geoffrey R. Spedding

\section{To cite this version:}

Patrice Meunier, Peter Diamessis, Geoffrey R. Spedding. Self-preservation in stratified momentum wakes. Physics of Fluids, 2006, 18, pp.10. 10.1063/1.2361294 . hal-00098367

\section{HAL Id: hal-00098367 https://hal.science/hal-00098367}

Submitted on 27 Jun 2007

HAL is a multi-disciplinary open access archive for the deposit and dissemination of scientific research documents, whether they are published or not. The documents may come from teaching and research institutions in France or abroad, or from public or private research centers.
L'archive ouverte pluridisciplinaire HAL, est destinée au dépôt et à la diffusion de documents scientifiques de niveau recherche, publiés ou non, émanant des établissements d'enseignement et de recherche français ou étrangers, des laboratoires publics ou privés. 


\title{
Self-preservation in Stratified Momentum Wakes
}

\author{
Patrice Meunier ${ }^{\dagger}$, Peter J. Diamessis ${ }^{2}$, and Geoffrey R. Spedding \\ Department of Aerospace \& Mechanical Engineering, \\ University of Southern California, Los Angeles, \\ CA 90089-1191, USA \\ †Institut de Recherche sur les Phénomènes Hors Équilibre, \\ 49 rue F. Joliot-Curie, F-13384 Marseille, France \\ 2 School of Civil and Environmental Engineering, \\ Cornell University, Ithaca, NY 14853, USA
}

(Dated: August 30, 2006)

\begin{abstract}
A general model is described for drag wakes in a linearly stratified fluid, based on the selfpreservation of the flow. It is assumed that the buoyancy-controlled self-similar wake expands in the horizontal direction due to turbulent diffusion and in the vertical direction due to viscous diffusion. The mean characteristics of the wake (height, width and velocity defect) are analytically derived and show good agreement with existing data from experimental and numerical results. Moreover, the three regimes previously found in the literature that characterise different dynamical phases of the wake evolution are recovered, and two new regimes are found. The model allows for prediction of characteristic length and velocity scales at the high Reynolds numbers of large-scale applications of geophysical and naval origin.
\end{abstract}




\section{INTRODUCTION}

Research on stratified wakes has both meteorological (flow over islands, mountains or sea-mounts) and naval applications (submerged bodies). In each case, the Reynolds number ( $R e=U_{B} D / \nu$, for an object of diameter $D$ in a uniform freestream $\left.U_{B}\right)$ is several orders of magnitude higher than obtained in both laboratory experiments and numerical simulations. By contrast, the appropriate internal Froude number $\left(F=2 U_{B} / N D\right.$, for a fluid of buoyancy frequency $N=\sqrt{-(g / \rho)(\partial \rho / \partial z)}$, which ranges from $10^{-1}$ to $10^{3}$, can be readily obtained experimentally and numerically. At sufficiently large values of Reynolds number and Froude number, the initial (near) wake is fully-turbulent in all three directions. Chomaz et al. [1] found the necessary criterion on $F$ to be $F \geq 4.5$, and a minimum value of $F \simeq 4$ was shown in scaling arguments [2] and later in experiments [3] where $F$ and $R e$ were varied independently. In [4] it was demonstrated that all wakes for $4 \leq F \leq 240$ have similar scaling behaviour, provided $R e$ is also sufficiently large (an approximate minimum value of $5 \times 10^{3}$ was suggested). Physically, the reason for the similar scaling is that since local wake velocities decrease, while local length scales increase, then a local Froude number based on these scales will decrease so that it becomes of order one, and thus the late stages of even a weakly stratified wake are eventually dominated by buoyancy effects.

The observations were consistent with previous experiments, simulations and analysis of decaying stratified turbulence, where buoyancy forces start to act at $N t \approx 1$ when the local dynamics experience the onset of buoyancy control (see Riley and Lelong [5] for a review). In experiments on self-propelled slender bodies, Lin and Pao [6] showed that buoyancy begins to affect the flow at $N t=2$, suspending the expansion of the wake in the vertical direction. This was followed by the emergence of large coherent structures as shown by dye pictures, which also showed that their aspect ratio (horizontal length scale compared with vertical extent) was large. The origin of these structures was later traced [7] to Kelvin-Helmholtz free shear-layer instabilities generated at the edge of the near wake and the gradual supression of vertical motions by the density gradient ensures that only these so-called pancake vortices 
persist into the late wake.

Ref. [4 found that two different regimes could be distinguished in the velocity field of the intermediate and late wakes. Initially, decay rates of horizontal mean velocity were found to be surprisingly low, with $U \sim N t^{-0.25}$ (instances where the defect velocity even increases have been reported [8]). There is then a transition to a steeper decay rate with exponents of approximately -0.76 , with the transition time apparently constant in buoyancy timescales of $N t \approx 50$. (While this transition time appeared to be independent of $F$ for the towed sphere experiments, a survey of the literature suggested large variation from $N t=10$ to $N t=180$ in the transition time between different laboratory or numerical experiments.) The subsequent steeper decay rate was maintained for all measurable times up to $N t \approx 2000$. The preceding intermediate regime is one where vertical velocities gradually become small, associated with radiation of internal waves from the wake to the ambient, and, it was conjectured, with conversion of potential to kinetic energy close to the wake centre. This adjustment period was termed the non-equilibrium regime (NEQ). The subsequent, late wake is characterised by the relative insignificance of vertical velocities and largely-horizontal motions account for almost all the remaining kinetic energy in the flow. The flow field however, is not two-dimensional (and its decay rate is not the same as for a two-dimensional wake, which would have a -0.5 exponent) because there is significant variability in the vertical, and the horizontal vorticity marking the shearing between neighbouring layers is stronger than the vertical vorticity [9]. This late wake regime was named the quasi-2D, or Q2D regime.

During both NEQ and Q2D, the wake width grows as $N t^{1 / 3}$ (or $x / D^{1 / 3}$ ), which is the same as would occur in a fully three-dimensional, unstratified turbulent wake. Since growth rates of vertical scales are very slow during NEQ, and since the stratified wake does not grow laterally faster than its $3 \mathrm{D}$ counterpart, momentum conservation requires that NEQ is associated with the observed low decay rates. Finally, it was argued by [4] that the very early wake at moderate to high $F$ would most likely evolve with buoyancy forces playing little part and so a sequence of 3D-NEQ-Q2D was identified as characterising stratified wakes.

The basic phenomenology of the NEQ and Q2D regimes has been replicated also in 
numerical simulations [10, 11], but agreement on vertical length scales has been more elusive. Chomaz et al. [12] found that the wake height increases very rapidly in the late wake, and a transition from almost zero growth to rapid growth (but different growth rates than [12]) of vertical length scales was reported in further experiments by Spedding [9]. Numerical simulations appeared to share similar features [10, 11], but the setting of the initial vertical length scales according to $F$ was never resolved as the simulations would typically be run for only one finite value of $F$. This vertical growth of the wake is not yet well understood and precise measurements have been difficult to obtain, both experimentally and numerically.

The experimental results were obtained for the particular case of the wake of a sphere, but were successfully extended to the general case of other bluff bodies [13], by considering the momentum thickness as the proper lengthscale rather than the body diameter. For an axisymmetric bluff body, the momentum thickness is defined as $D_{\mathrm{m}}=D \sqrt{c_{D} / 2}$, where $c_{D}$ is the drag coefficient for the bluff body in an unstratified fluid. In fact, the very same wake scalings could be extended to almost all wakes generated by propelled bluff bodies [14] (including all those of practical interest), if the momentum thickness is defined as $D_{\mathrm{m}}=D \sqrt{c_{D} / 2} \sqrt{1-U_{C}^{2} / U_{B}^{2}}$, where $U_{C}$ is the particular velocity of the self-propelled bluff body for which the wake becomes momentumless. The relevant non-dimensional parameters become a momentum Reynolds number $R e_{\mathrm{m}}=U_{B} D_{\mathrm{m}} / \nu$ and a momentum Froude number $F_{\mathrm{m}}=2 U_{B} / N D_{\mathrm{m}}$

The empirical scaling laws describing the velocity defect and wake width as developed in [3, 4, 13, 14] apply to any bluff body wake or jet in a uniform, stable density gradient. However, they remain empirical findings, linked by incomplete arguments and a coherent theoretical framework has not been proposed. Moreover, compared with practical applications where Reynolds numbers are usually in the range of $10^{6}-10^{8}$, the limited range of $R e$ of the experiments upon which they are based makes the extrapolation of these scaling arguments to higher $R e$ somewhat speculative. The laboratory experiments show no significant Re dependence over $R e \in\left[5 \times 10^{3}, 2.8 \times 10^{4}\right]$ and the largest $R e$ in numerical experiment was $10^{5}$, indicating that the results might at least be only weakly $R e$-dependent. On the 
other hand, high $R e$, high resolution, numerical simulations of a simple stratified flow geometry [15] have indicated that as $R e$ increases, more small-scale instabilities of the vertical shear layers develop, becoming a continuous source of small-scale turbulence, and raising the idea that at sufficiently high $R e$, the large-scale coherent structures seen in laboratory experiment may never develop, or survive. However in the range of $R e_{l} \in[800,6400]$, where $l$ is a numerically-imposed initial length scale of the Taylor-Green vortices, the large-scales were only weakly dependent on $R e_{l}$, a finding that is consistent with the increased fine structure of the higher Re wake simulations of Dommermuth et al. [11], but which still produced large-scale structures in the late wake.

In this paper, some scaling relations are derived, based on the evolution of self-similar solutions of reduced equations of motion for stratified momentum wakes with high Reynolds number and high Froude number. Analytical results will be derived for the horizontal and vertical length scales, and for the mean and turbulence profiles. The agreement with existing data from laboratory and numerical experiment will be shown to be good, and the scaling behaviour for high Reynolds number can be clearly shown.

\section{A SELF-PRESERVING THEORY}

\section{A General equations}

We will suppose that the fluid is linearly stratified with a constant buoyancy frequency $N$ and a density $\rho_{0}$ at the bluff body's height, and write the Navier-Stokes equations in the Boussinesq approximation:

$$
\frac{\partial \mathbf{u}}{\partial t}+(\mathbf{u} \cdot \nabla) \mathbf{u}=\frac{-\nabla p}{\rho_{0}}+\nu \triangle \mathbf{u}+\frac{\rho}{\rho_{0}} \mathbf{g}
$$

together with the incompressibility condition:

$$
\nabla \mathbf{u}=0
$$


and the equation for the evolution of the density

$$
\frac{\partial \rho}{\partial t}+\mathbf{u} \cdot \nabla \rho=\kappa \Delta \rho
$$

Since the flow is stationary in the frame of reference moving with the bluff body, $U=U(y, z)$, the velocity can be decomposed as a temporal mean and a fluctuating part $\mathbf{u}=U+\mathbf{u}^{\prime}$. The mean velocity is equal to the bluff body velocity $U_{B}$ in the far field, and the wake has a defect velocity of the order of $U_{0}$, as indicated schematically on figure 1. We then follow the standard analysis for the turbulent wake in a non-stratified flow, found for example in Tennekes and Lumley [16]. By averaging the streamwise component of the momentum equation (1), one arrives at:

$$
U \frac{\partial U}{\partial x}+V \frac{\partial U}{\partial y}+W \frac{\partial U}{\partial z}+\frac{\partial\left\langle u^{\prime 2}\right\rangle}{\partial x}+\frac{\partial\left\langle u^{\prime} v^{\prime}\right\rangle}{\partial y}+\frac{\partial\left\langle u^{\prime} w^{\prime}\right\rangle}{\partial z}=-\frac{1}{\rho_{0}} \frac{\partial P}{\partial x}+\nu \triangle U
$$

Note that on this component of velocity, stratification effects are in fact absent, except insofar as they would lead to non-zero mean values of $\left\langle u^{\prime} w^{\prime}\right\rangle$. This is a consequence of assuming $w$ to be small at late times, and thus retaining only the streamwise momentum components. Now, following [16], we assume that the flow is nearly parallel, so the lengthscale $l$ in the $y$ and $z$ direction is smaller than the lengthscale $L$ in the $x$ direction. Since the focus is on late wake evolution, we may also assume that the velocity amplitude $U_{0}$ is much smaller than the tow speed $U_{B}$, which is valid at least far from the bluff body. The second, third and fourth terms in eq. (4) can therefore be neglected since they are of order $U_{0}^{2} / L$, which is $U_{0} / U_{B}$ smaller than the first term. The pressure term can be shown to be of the order of $\partial\left\langle v^{2}\right\rangle / \partial x$ by inspection of the leading order terms in the cross-stream momentum equation, which are again negligible with respect to the first term. At high Reynolds numbers, the viscous terms could be entirely neglected at early stages, but will be retained because they will reappear later, as the local Reynolds number decreases in time. Here though, the term $\partial^{2} U / \partial x^{2}$ is small compared with $\partial^{2} U / \partial y^{2}$ and may be omitted. Finally, the mean velocity $U$ can be replaced by $U_{B}$ in the first term, which leads to a simple form for the streamwise 
momentum equation:

$$
U_{B} \frac{\partial U}{\partial x}=-\frac{\partial\left\langle u^{\prime} v^{\prime}\right\rangle}{\partial y}-\frac{\partial\left\langle u^{\prime} w^{\prime}\right\rangle}{\partial z}+\nu\left(\frac{\partial^{2} U}{\partial y^{2}}+\frac{\partial^{2} U}{\partial z^{2}}\right)
$$

It has been shown experimentally that mean profiles are close to Gaussian in both nonstratified wakes [16] and also in stratified wakes [3, 4], and an example of the latter is shown in Fig. 2(a) from [17]. Thus we may search for Gaussian solutions to eq.(5), of the form

$$
U=U_{B}-U_{0} \exp \left(-\frac{y^{2}}{2 L_{y}^{2}}-\frac{z^{2}}{2 L_{z}^{2}}\right),
$$

where the velocity defect $U_{0}$, the wake width $L_{y}$ and height $L_{z}$ are functions of $x$ only. Other solutions might be found [18], but this Gaussian solution is convenient since it is the simplest self-similar solution for a momentum wake.

Under the Boussinesq approximation, the momentum flux $J=\iint \rho U_{B}\left(U-U_{B}\right) d y d z$ is equal to $\rho_{0} \iint U_{B}\left(U-U_{B}\right) d y d z$. By integrating (5) on a transverse section, the mean momentum flux can be shown to be independent of the position $x$ and equal to the drag force $F_{D}=c_{D} \rho_{0} U_{B}^{2} \pi D^{2} / 8$, where $c_{D}$ is the body drag coefficient. Thus by defining the momentum thickness as $D_{\mathrm{m}}=D \sqrt{c_{D} / 2}$, the integration of (6) leads to a relation between $U_{0}, L_{y}$ and $L_{z}$ :

$$
U_{0} L_{y} L_{z}=U_{B} D_{\mathrm{m}}^{2} / 8
$$

\section{B Initial three-dimensional regime}

At high Froude numbers, the stratification is negligible in the early stages and the flow may be expected to be three-dimensional and the mean velocity to be axisymmetric. It is common practice to suppose that the Reynolds stress is proportional to the mean shear $\left(\left\langle u^{\prime} v^{\prime}\right\rangle=-\nu_{3} \partial U / \partial y\right)$, with an eddy viscosity $\nu_{3}$ independent of $y$ and $z$ in the 3D regime. Partial experimental support for this notion has been found in [13, 14] for stratified wakes. Based on the mixing length model, or based on dimensional grounds, the eddy viscosity should be proportional to $U_{0} L_{y}$, which defines a turbulent Reynolds number $R_{3}=U_{0} L_{y} / \nu_{3}$ 
for the 3D regime. Bevilaqua \& Lykoudis [19] found

$$
R_{3}=4
$$

in the case of the sphere, for the same definitions of the mean profile, and this value will be used in the following.

We will also assume that the Reynolds number is sufficiently high to neglect the viscous terms in the $3 \mathrm{D}$ stage (it will be shown to be true when $F<<R e^{3}$ ). The momentum equation thus becomes a standard diffusion equation,

$$
U_{B} \frac{\partial U}{\partial x}=\frac{U_{0} L_{y}}{R_{3}} \frac{\partial^{2} U}{\partial y^{2}}+\frac{U_{0} L_{z}}{R_{3}} \frac{\partial^{2} U}{\partial z^{2}}
$$

except that the diffusion coefficient $U_{0} L_{y} / R_{3}$ is now $x$-dependent. Imposing a Gaussian form of the mean profile (6) into (8), leads to

$$
\frac{\dot{U}_{0}}{U_{0}}+\frac{y^{2}}{L_{y}^{2}} \frac{\dot{L_{y}}}{L_{y}}+\frac{z^{2}}{L_{z}^{2}} \frac{\dot{L_{z}}}{L_{z}}=\frac{U_{0}}{R_{3} L_{y} U_{B}}\left(\frac{y^{2}}{L_{y}^{2}}-1\right)+\frac{U_{0}}{R_{3} L_{z} U_{B}}\left(\frac{z^{2}}{L_{z}^{2}}-1\right),
$$

where the dot denotes the derivative with respect to the downstream distance $\left(\dot{U}_{0}=d U_{0} / d x\right)$.

The Gaussian form introduced in (6) is a solution of the Navier-Stokes equations if and only if eq. (9) is valid for any $y$ and $z$. Since this equation only contains 3 types of terms (terms in $y^{2}$, terms in $z^{2}$ and terms independent of $y$ and $z$ ), (6) is a solution if and only if their three coefficients vanish, which leads to three independent equations:

$$
\begin{gathered}
\dot{L_{y}}=\frac{U_{0}}{R_{3} U_{B}} \\
\dot{L_{z}}=\frac{U_{0}}{R_{3} U_{B}} \\
\frac{\dot{U}_{0}}{U_{0}}=-\frac{U_{0}}{R_{3} L_{y} U_{B}}-\frac{U_{0}}{R_{3} L_{z} U_{B}}
\end{gathered}
$$

Since there are three variables $U_{0}, L_{y}$ and $L_{z}$, a Gaussian solution can exist. By summing (10) $\left./ L_{y}+11\right) / L_{z}+12$, we recover that $U_{0} L_{y} L_{z}$ is constant, as was found using the momentum flux (7). Then, introducing this condition (7) into (10) and (11), we also recover the 
solution given by Tennekes and Lumley[16]:

$$
\begin{gathered}
L_{y}=L_{z}=D_{\mathrm{m}}\left(\frac{3 x}{8 R_{3} D_{\mathrm{m}}}\right)^{1 / 3} \\
U_{0}=\frac{U_{B}}{8}\left(\frac{3 x}{8 R_{3} D_{\mathrm{m}}}\right)^{-2 / 3}
\end{gathered}
$$

This solution is valid when the local Froude number (defined as $U_{0} / N L_{y}$ ) is larger than one, which is met only close to the bluff body. Indeed, this local Froude number decreases as $1 / x$ and reaches unity for $x_{I} / D_{\mathrm{m}}=R_{3} F_{\mathrm{m}} / 6 \approx 0.66 F_{\mathrm{m}}$. This transition distance is in good agreement with previous experimental results [6, 4], where it was shown, or inferred, that the wake is $3 \mathrm{D}$ until $N t \approx 2$, i.e. until $x_{I} / D_{\mathrm{m}}=F_{\mathrm{m}}$ in our units. We may also note that since the form of the reduced equations (5) and the presumed existence of self-similar solutions (6) requires that $x / D$ is large, then the simultaneous requirement of large $x / D$ and large local $F$ limits this model system to flows with large initial $F$. Only then can a self-similar flow be imagined before the transition distance, $x_{I}$. In fact, the exact value of this transition distance very weakly influences the late stages and we will assume that the transition occurs for $x_{I} / D_{\mathrm{m}}=F_{\mathrm{m}}$. The vertical width of the wake at this point is thus:

$$
L_{v}=D_{\mathrm{m}}\left(\frac{3 F_{\mathrm{m}}}{8 R_{3}}\right)^{1 / 3}
$$

This classical solution, with no stratification effects, will now be extended into the stratified regime, which occurs for $x$ larger than $x_{I}$.

\section{Stratified regime}

In the late wake, vertical velocity fluctuations eventually subside as one component of the wake disturbance propagates away in the form of internal waves [17], leaving residual motions whose time evolution is much slower [20, 21]. Consequently the vertical Reynolds stress $\left\langle u^{\prime} w^{\prime}\right\rangle$ becomes much smaller than the horizontal Reynolds stress $\left\langle u^{\prime} v^{\prime}\right\rangle$ in the far-wake, strongly-stratified regime. The experimental results of Fig. 2(b) compare these two terms at 
$N t=44$ and show that the first term is about an order of magnitude smaller than the second. As a convenient simplifying assumption, valid at least for the far wake, we will therefore neglect the vertical Reynolds stress in the averaged momentum equation (5). However, we should note how neglecting $\left\langle u^{\prime} w^{\prime}\right\rangle$ removes much physics from this intermediate regime. All contributions from internal wave motions which lead to significant peaks in experimental measurements of $\left\langle u^{\prime} w^{\prime}\right\rangle$ as late as $N t=15$ [17] are omitted, as are any production terms where energy is drawn to the mean flow from the turbulent motions. Anisotropy of the dissipation rate tensor and of the velocity and vorticity energy balance has been noted and discussed in simulations of stratified turbulence[22, 23] and specifically for DNS of stratified wakes[10, 11]. The analysis that follows must be considered one where such intermediate dynamics are ignored, valid at the latter stages of the wake evolution only when $w \approx 0$ and $\left\langle u^{\prime} w^{\prime}\right\rangle<\left\langle\left\langle u^{\prime} v^{\prime}\right\rangle\right.$. It will prove instructive to see how much (or how little) of the mean flow dynamics can be found with this strong simplification.

We may recall that one of the purposes of this model is to find simplified expressions that both respect current results from laboratory and computational work, and at the same time give explicit predictions on how decaying turbulence scales at the much higher Reynolds numbers characteristic of ocean conditions. Higher Reynolds number simulations are just now becoming available, and some [see 15] raise the question as to whether the pancake vortices may be unstable with respect to the mean shear. (See also Fig. 5(c) of Waite \& Bartello[24] for small scale instabilities at moderate stratifications.) These instabilities (Kelvin-Helmholtz) could lead to non-zero vertical Reynolds stresses, and if this is shown to be the case then the strong assumption of $\left\langle u^{\prime} w^{\prime}\right\rangle<\left\langle\left\langle u^{\prime} v^{\prime}\right\rangle\right.$ will need to be modified.

The turbulent motion in the horizontal direction will continue to be modelled as a turbulent diffusion and so we will continue to assume that the horizontal Reynolds stress is proportional to the mean shear $\left(\left\langle u^{\prime} v^{\prime}\right\rangle=\nu_{2} \partial U / \partial y\right)$, consistent with available experimental evidence (see Fig. 2b). The eddy viscosity is therefore now characterized by a two-dimensional Reynolds number $\left(R_{2}=U_{0} L_{y} / \nu_{2}\right)$. It is two-dimensional because the supression of vertical velocities by the stratification at late times ( $N t \geq 40$, for example) has allowed us to assume 
that $\left\langle u^{\prime} w^{\prime}\right\rangle<\left\langle\left\langle u^{\prime} v^{\prime}\right\rangle\right.$. This flow regime is denoted BC for buoyancy-controlled, where the effect of the stratification is supposed to be sufficiently strong that the averaged equations of motion in a horizontal plane have no explicit buoyancy terms in them. Note that by neglecting the buoyancy terms entirely, this approach differs from the usual scaling expansion where $F<<1$ (e.g. [20, 25, 26]).

In late wakes of towed bluff bodies in stratified fluids $R_{2}$ was found to be close to 15 , regardless of initial conditions (within 30\%) [13], so this numerical value is retained in the following. By neglecting the vertical Reynolds stress while retaining the horizontal Reynolds stress in (5), we reach again a standard diffusion equation for the buoyancy-controlled flow,

$$
U_{B} \frac{\partial U}{\partial x}=\left(\frac{U_{0} L_{y}}{R_{2}}+\nu\right) \frac{\partial^{2} U}{\partial y^{2}}+\nu \frac{\partial^{2} U}{\partial z^{2}} .
$$

As in the 3D regime, a Gaussian solution is introduced (6) into (16), which leads to an equation with only terms in $y^{2}$, terms in $z^{2}$ and terms independent of $y$ and $z$. The Gaussian form (6) is thus a solution if and only if the three coefficients of these terms vanish, corresponding to the three following equations:

$$
\begin{gathered}
\dot{L_{y}}=\frac{U_{0}}{R_{2} U_{B}}+\frac{\nu}{U_{B} L_{y}} \\
\dot{L_{z}}=\frac{\nu}{U_{B} L_{z}} \\
\frac{\dot{U}_{0}}{U_{0}}=-\frac{U_{0}}{R_{2} L_{y} U_{B}}-\frac{\nu}{U_{B} L_{y}^{2}}-\frac{\nu}{U_{B} L_{z}^{2}}
\end{gathered}
$$

As before, the conservation of the momentum flux is recovered (7) by summing (10) $/ L_{y}+11 / L_{z}+12$. The equation for the vertical wake width (18) is characteristic of a laminar diffusion and can be integrated by setting initial conditions $L_{z}=L_{v}$ at $x / D_{\mathrm{m}}=F_{\mathrm{m}}$ to arrive at

$$
L_{z}=\sqrt{L_{v}^{2}+\frac{2 D_{\mathrm{m}}^{2}}{R e_{\mathrm{m}}}\left(x / D_{\mathrm{m}}-F_{\mathrm{m}}\right)} .
$$


This solution is plotted in Fig. 3(a) as a dotted line for $x / D_{\mathrm{m}}>F_{\mathrm{m}}$, together with the non-stratified solution 13 for $x / D_{\mathrm{m}}<F_{\mathrm{m}}$. For small $x / D, L_{z} \approx L_{v}$ in eq.20 and vertical growth is at first negligible. The second term eventually becomes larger than $L_{v}^{2}$ (i.e. at sufficiently large $x / D$ ), and when these two terms in the square root of eq.20 are of equal magnitude, a second transition point can be identified at a downstream distance of the order of the Reynolds number:

$$
x_{I I} / D_{\mathrm{m}}=\frac{R e_{\mathrm{m}}}{2}\left(\frac{3 F_{\mathrm{m}}}{8 R_{3}}\right)^{2 / 3} .
$$

The wake height then increases as $\sqrt{x}$ due to viscous diffusion as the wake enters the quasi-2D regime (Q2D). It can be noted that for higher Reynolds numbers, the NEQ and buoyancycontrolled $(\mathrm{BC})$ regimes will last longer. Since the NEQ regime in particular is associated with quite rich dynamics and complex interactions between internal waves and turbulence, the implication is that at full scale, these complex fields will assume even more importance in turbulence evolution in a stratified ambient. The possible consequences in geophysical and naval applications will be discussed in the conclusion.

To calculate the horizontal wake width, we introduce (7) into (17), which leads to:

$$
L_{y} \dot{L}_{y}=\frac{D_{\mathrm{m}}^{2}}{8 L_{z} R_{2}}+\frac{\nu}{U_{B}}
$$

This can be integrated by introducing the expression of the vertical wake width $(20)$, with initial conditions $L_{y}=L_{v}$ at $x / D_{\mathrm{m}}=F_{\mathrm{m}}$ :

$$
L_{y}=\left[L_{v}^{2}+\frac{D_{\mathrm{m}} R e_{\mathrm{m}}}{4 R_{2}}\left(\sqrt{L_{v}^{2}+\frac{2 D_{\mathrm{m}}^{2}}{R e_{\mathrm{m}}}\left(\frac{x}{D_{\mathrm{m}}}-F_{\mathrm{m}}\right)}-L_{v}\right)+\frac{2 D_{\mathrm{m}}^{2}}{R e_{\mathrm{m}}}\left(\frac{x}{D_{\mathrm{m}}}-F_{\mathrm{m}}\right)\right]^{1 / 2}
$$

The solution is plotted in Fig. 3(a) as a dash-dotted line. In the BC regime $\left(x_{I}<x<\right.$ $x_{I I}$ ), the horizontal wake width increases asymptotically (when $F_{\mathrm{m}}<<x / D_{\mathrm{m}}<<R e_{\mathrm{m}}$ ) as $\sqrt{x / L_{v} R_{2}} D_{\mathrm{m}} / 2$, with the same power law that was found theoretically for a two-dimensional non-stratified wake [16]. This regime is entered only after passing through the NEQ phase, which has been discovered experimentally [4, 8]. It appears here not as a regime defined explicitly, but as a transient between $3 \mathrm{D}$ and BC stages (note however, that this is simply a 
consequence of modelling decisions made initially). This transient stage lasts until $x / D_{\mathrm{m}}=$ $50 F_{\mathrm{m}}$, independent of the Reynolds number. The BC regime ends at $x_{I I}$ defined previously, giving rise to the quasi-2D regime $(\mathrm{Q} 2 \mathrm{D})$, for which the wake width increases more slowly as $x^{1 / 4}: L_{y} \sim\left(R e_{\mathrm{m}} x / 2 D_{\mathrm{m}}\right)^{1 / 4} D_{\mathrm{m}} / \sqrt{2 R_{2}}$. This approximation can be obtained by a Taylor expansion of (22) assuming $x_{I}<<x_{I I}<<x$. Finally, at very late stages, a new regime appears, which we denote the viscous $3 \mathrm{D}$ regime (V3D), since now the wake width also increases by viscous diffusion as $\sqrt{2 \nu x / U_{B}}$. This regime starts for downstream distances larger than $x_{I I I}$, defined as

$$
x_{I I I} / D_{\mathrm{m}}=\frac{R e_{\mathrm{m}}^{3}}{32 R_{2}^{2}},
$$

which is obtained when the second and third terms inside the square root of eq. 22. are equal, i.e. when turbulent diffusion in the horizontal is accompanied by equal or greater molecular diffusion. The flow is expected to be laminar and axisymmetric, since the horizontal and vertical wake width become equal again. It was assumed in the model that the stratified effects would occur before the viscous effects, which is valid if $x_{I}<<x_{I I I}$, i.e. when $F<<R e^{3}$, which is easily met even for moderate Reynolds numbers.

Finally, the theory gives a prediction for the velocity defect $U_{0}$, by introducing $(20)$ and (22) into (7):

$$
\begin{aligned}
U_{0}= & \frac{U_{B}}{8}\left[\frac{L_{v}^{2}}{D_{m}^{2}}+\frac{2}{R e_{\mathrm{m}}}\left(\frac{x}{D_{\mathrm{m}}}-F_{\mathrm{m}}\right)\right]^{-1 / 2} * \\
& {\left[\frac{L_{v}^{2}}{D_{m}^{2}}+\frac{R e_{\mathrm{m}}}{4 R_{2}}\left(\sqrt{\frac{L_{v}^{2}}{D_{m}^{2}}+\frac{2}{R e_{\mathrm{m}}}\left(\frac{x}{D_{\mathrm{m}}}-F_{\mathrm{m}}\right)}-L_{v}\right)+\frac{2}{R e_{\mathrm{m}}}\left(\frac{x}{D_{\mathrm{m}}}-F_{\mathrm{m}}\right)\right]^{-1 / 2} }
\end{aligned}
$$

This solution is plotted in Fig. 3(b). In the BC stage, the velocity defect decreases as $U_{B} D_{\mathrm{m}} \sqrt{R_{2} / x L_{v}} / 4$. This state is reached after a transient (at least in this framework) stage (the NEQ regime) in which the velocity decreases very slowly. The BC regime is followed by the Q2D regime, in which the velocity defect scales as $x^{-3 / 4}$ : $U_{0} \sim \sqrt{R_{2}}\left(2 R e_{\mathrm{m}}\right)^{1 / 4}\left(x / D_{\mathrm{m}}\right)^{-3 / 4} U_{B} / 8$. This regime ends at $x_{I I I} / D_{\mathrm{m}}$, and the wake then enters the viscous $3 \mathrm{D}$ regime $(\mathrm{V} 3 \mathrm{D})$, in which the velocity defect decreases even faster: 
$U_{0} \sim\left(U_{B} D_{\mathrm{m}}\right) /\left(16 R e_{\mathrm{m}} x\right)$. This regime has never been observed experimentally as it occurs for very far downstream distances.

The theory outlined above gives a universal prediction for wake width, height and velocity defect in a stratified wake given by (22), 20) and (24). The model recovers the approximate scaling behaviour of the experimentally-determined 3D-NEQ-Q2D progression from fully three-dimensional to quasi two-dimensional (strongly stratified) flow. It also predicts a Reynolds number dependence in transition lengths between these regimes, and in particular, the NEQ regime extends into a strongly buoyancy-controlled (BC) regime, where the dominating influence of the stratification is to constrain the flow to evolve in two-dimensional layers, when the stratification then, paradoxically, has no direct influence. At the end of the Q2D regime there appears a viscous 3D regime (V3D), in which the wake becomes laminar and grows in all directions by diffusion only. Neither the BC, nor the V3D regimes have been observed yet, because experiment Reynolds numbers and observation times have not been large enough. The model predictions can now be compared with laboratory and numerical experiment for specific values of the Reynolds and Froude number.

\section{COMPARISON WITH EXPERIMENT}

\section{A Variations with the downstream distance}

The mean characteristics of the wake predicted by the model were plotted in Fig. 3 at a high Reynolds number, and for a large range of downstream distances. This was designed to define clearly the different regimes and to show their asymptotic behaviours. We will now focus on a common Froude number and Reynolds number pair encountered in the literature, to compare the model to data from both laboratory and numerical experiment. A momentum Froude number of 8.89, and a momentum Reynolds number of 2250 correspond to $F=4$ and $R e=5000$ for the canonical towed-sphere example, allowing comparisons with experimental results from three different facilities [12, 4, 8] and with numerical simulations 
that used three different methods [10, 11, 27].

First, we compare the horizontal wake width $L_{y}$ in Fig. 4. All experimental and numerical data fall on the model within $30 \%$. The model is thus seemingly in good agreement with the data, even though it does not contain any fitting parameter. Note however, that the experimental growth rates (over $4 \leq F \leq 240$ and $4.7 \leq R e \leq 10.6 \times 10^{4}$ ) have a constant power law exponent of $0.35 \pm 0.03$, which is different from both the early (NEQ/BC: $x^{1 / 2}$ ) and later (Q2D: $x^{1 / 4}$ ) time exponents of the model. The reason for the apparent agreement in Fig. 3 is $R e$ is low enough for the NEQ and BC regimes to be barely distinguishable. The mixed result of exponents between the two ( 0.5 and 0.25 respectively), combined with the initial phase of $\mathrm{Q} 2 \mathrm{D}$ in the theory gives a result that looks like the data. The prediction is that if $R e$ is high enough (for example, in a field measurement), then the separate regimes will be observed.

The vertical wake height $L_{z}$ is compared in Fig. 5. This measurement is hard to achieve experimentally, and there is an observed dependence of initial wake height on $F$, which is not taken into account in this figure. The results included here of Chomaz et al. [12] were re-analysed and plotted by assuming that the mean profiles were Gaussian so as to calculate an equivalent quantitative value of the wake height. Although these particular results do not all fall within the agreement of the other experimental data (at least at moderate $x / D$ ), the slope is in fact quite consistent with a purely viscous diffusion growth process, and not significantly accelerated, as seemed to be the case when shown in their original form([12], Fig. 9). In general, both numerical and experimental results show a large plateau, followed by a steep increase at later stages. This corresponds to the NEQ and BC regimes in which the wake height is constant, followed by the Q2D regime in which the wake height increases by viscous diffusion as $\sqrt{x}$. This basic phenomenon therefore requires no more complex an explanation than initial suppression of vertical growth by stratification, followed by viscous diffusion in the vertical at later times, as specified in the model problem. However, it is also important to note that considerable differences persist at late times (Q2D), and that any $F$-dependence in NEQ/BC remains unaccounted for in this plot. 
The mean velocity defect $U_{0}$ is compared in Fig. 6. In experiment (dotted line in Fig. 6), the initial decay rate is imagined to be $x^{-2 / 3}$ as for an unstratified wake, followed by the non-equilibrium period when measured decay rates are significantly lower $x^{-0.25 \pm 0.04}[4]$. (Measurements by Bonnier and Eiff [8] in fact show a possible increase in $U_{0}$ at early-NEQ, as shown by the crosses for one of their experiments.) In the model, NEQ is supposed to last from $x / D_{\mathrm{m}}=F_{\mathrm{m}}$ to $x / D_{\mathrm{m}} \approx 50 F_{\mathrm{m}}$. In experiment, NEQ was found to begin at $N t \approx 2$ and end at $N t \approx 50$ and the equivalent $x / D$ varies with $F($ as $x / D=N t . F / 2)$. $U_{0}$ then decreases more rapidly, as $x^{-0.75}$, which is characteristic of the Q2D regime: in experiments, the decay exponent was found to be $-0.76 \pm 0.12$. The agreement between model predictions (solid line) and laboratory (dotted line, crosses) and numerical (open symbols) experiment is good given that all numerical and experimental measurements fall within $50 \%$ of the model prediction. Again, the NEQ and BC regimes of the model are very close at this $R e$, and the net effect is to mimic the experimental results reasonably well. The collapse of points in the Q2D regime is also good. Fig. 6 can be viewed as a consequence of momentum conservation, given Figs 4 and 5 , and just as any $F$-dependence is omitted from Fig. 5, so it is also absent in Fig. 6.

The following section considers the $F$ and $R e$ dependence in the model and experimental data in more detail.

\section{B Dependence on the Froude and the Reynolds number}

By varying the Froude number over almost two decades, Spedding [4] showed that the velocity defect scales as $(N t)^{-0.76} F^{-2 / 3}$ at the beginning of the Q2D regime. This dependence on the Froude number can be recovered theoretically by looking at the velocity defect in the BC regime, where $U_{0} \sim 1 / \sqrt{x L_{v}} \sim(N t)^{-1 / 2} F^{-2 / 3}$. The dependence differs slightly in the Q2D regime $\left(U_{0} \sim(N t)^{-3 / 4} F^{-3 / 4}\right)$, but such variations might be difficult to distinguish in practice.

The primary feature of stratified wakes is that the wake height saturates at a specific 
vertical lengthscale $L_{v}$, which can be readily deduced from experimental and numerical results by looking at the value of the initial plateau of the wake height. These values are compared in Fig. 7 with the theoretical prediction given by Eq. (15). The model predicts that $L_{v}$ scales as $F^{1 / 3}$, while it was found to vary as $F^{0.6}$ experimentally [9]. The discrepancy is perhaps unsurprising, given the complete neglect of physical mechanisms involving any correlation of vertical velocities, density field structure and internal wave dynamics. Further improvements in the model will be especially important at high Froude number, for example, where $L_{v}$ is underestimated by a factor 2 . Although the numerical simulations show good agreement, they do not predict the variation of $L_{v}$ with the Froude number, since they were always performed at low $F$. Moreover, it is far from a trivial matter knowing how a numerical simulation should be initialised with correct initial length scales where any $L_{v}$ could be truly claimed to be an independent outcome of the model, rather than an input.

Finally, we can determine the downstream distance $x_{I I}$ at which the transition between the $\mathrm{BC}$ and the $\mathrm{Q} 2 \mathrm{D}$ regime occurs. It is plotted in Fig. 8 as a function of the Froude and the Reynolds number. While the general dependence of $x_{I I}$ as $R e_{\mathrm{m}} F_{\mathrm{m}}^{2 / 3}$ is consistent with the data, for any given $R e_{\mathrm{m}}$ or $F_{\mathrm{m}}$, the model always overestimates $x_{I I}$, which again shows that the model might be improved. This might include improved modeling and/or higher order correction of terms leading to vertical transport, or some way of accounting for initial conditions whose effect could be felt out to $x_{I I}$. Further experiments and numerical simulations over a broad range of $R e$ and run for long times might help to point out ways in which this can be done.

\section{CONCLUSIONS}

The general theory developed here predicts the mean profile evolution of a drag wake in a linearly stratified fluid. The model is based on an assumption of eddy viscosity in the horizontal direction, which allows the mean momentum equations to be closed by assuming that the horizontal Reynolds stress is proportional to the mean shear. The (constant) value 
of the eddy viscosity is chosen from relevant experiments in stratified wakes, and not as an $a$ posteriori fitting parameter. The evolution of the wake in the vertical is modelled by viscous diffusion alone for $N t>1$, which is reasonable for the moderate Re in laboratory experiments and numerical simulations. In this respect, the approach is analagous to stratified dipole and turbulence evolution experiments where experimental data were quite closely matched by numerical models with turbulent and viscous diffusion in the horizontal and vertical, respectively. [28, 29] The averaged equations of motion show that a Gaussian solution can be found and allow an analytical determination of the wake height, the wake width and the velocity defect.

Existing results from the literature for wake height, wake width and velocity defect, from both laboratory and numerical experiment, agree with the theoretical predictions within $50 \%$. The three different regimes found in the literature that characterise decaying stratified flows (3D, NEQ and Q2D regimes) are recovered, and two new regimes are proposed: a buoyancy-controlled $(\mathrm{BC})$ regime which should be observed (as distinct from the NEQ regime) at very high Reynolds numbers, and a viscous 3D regime, which should be observed at very late stages. Note that since the formulation involves self-similar solutions of simplified equations of motion, transients at relatively small $x / D$, such as the acceleration phase noted by [8], are not accounted for.

These results apply to any self-similar shear flow in a stratified fluid, where a momentum thickness is defined by $D_{\mathrm{m}}=D \sqrt{c_{D} / 2}$. Such a model can even be extended to the majority of cases of self-propelled bodies [14], whenever the wake momentum balance departs from zero by more than $2 \%$; that will be the case for even small accelerations, whenever there is significant wave drag, and whenever the ambient is non-uniform.

The extension to high Reynolds numbers is of interest to many practical geophysical and naval applications. Examples include large-scale submerged bodies and atmospheric wakes of mountains or oceanic wakes of islands and sea-mounts. The mean characteristics of the wake are given analytically by the model in equations $(20),(22)$ and $(24)$ and the previous experimental and numerical results can be extrapolated to high Reynolds numbers using the 
similarity laws of the model.

One of the principal consequences is that the Q2D regime appears later for higher Reynolds numbers (at $x / D=R e F^{2 / 3}$, or $N t=R e F^{-1 / 3}$ ). Since the mean velocity decay rates in the intervening $\mathrm{NEQ}$ and $\mathrm{BC}$ regime are lower than in either the surrounding 3D or Q2D regimes, this scaling behaviour predicts that the high magnitude defect wakes that have been observed in laboratory experiment should be even more evident with higher Re. In this regard, the model described in this paper bears a certain similarity with another, spectrally based, theoretical model of localized stratified turbulence, [30] and despite fundamental differences in their formulation, both models identify a signature of the initial $R e$ on the intermediate-to-late-time flow dynamics, which persists for longer times with increasing Re.

The model could be extended to more specialised cases, such as bluff body wakes with an angle of attack[31], and to the particular case of exactly momentumless wakes. In both cases, the mean profiles are not simple Gaussians and it would be interesting to test other scaling functions.

The support of ONR Grant no. N00014-04-1-0034 administered by Dr. R. Joslin is most gratefully acknowledged.

\section{REFERENCES}

[1] J. M. Chomaz, P. Bonneton, and E. J. Hopfinger, The Structure of the Near Wake of a Sphere Moving Horizontally in a Stratified Fluid, J. Fluid Mech. 254, 1 (1993b).

[2] G. R. Spedding, F. K. Browand, and A. M. Fincham, The long-time evolution of the initially-turbulent wake of a sphere in a stable stratification, Dyn. Atmos. Ocean 23, 171 (1996a).

[3] G. R. Spedding, F. K. Browand, and A. M. Fincham, Turbulence, Similarity Scaling and Vortex Geometry in the Wake of a Towed Sphere in a Stably Stratified Fluid, J. Fluid Mech. 314, 53 (1996b). 
[4] G. R. Spedding, The Evolution of Initially Turbulent Bluff-Body Wakes at High Internal Froude Number, J. Fluid Mech. 337, 283 (1997).

[5] J. R. Riley and M. P. Lelong, Fluid Motions in the Presence of Strong Stable Stratification, Ann. Rev. Fluid Mech. 32, 613 (2000).

[6] J. T. Lin and Y. H. Pao, Wakes in Stratified Fluids: a Review, Ann. Rev. Fluid Mech. 11, 317 (1979).

[7] G. R. Spedding, The Streamwise Spacing of Adjacent Coherent Structures in Stratified Wakes, Phys. Fluids 14(11), 3820 (2002).

[8] M. Bonnier and O. Eiff, Experimental investigation of the collapse of a turbulent wake in a stably stratified fluid, Phys. Fluids 14(2), 791 (2002).

[9] G. R. Spedding, Vertical structure in stratified wakes with high initial Froude number, J. Fluid Mech. 454, 71 (2002).

[10] M. J. Gourlay, S. C. Arendt, D. C. Fritts, and J. Werne, Numerical modeling of initially turbulent wakes with net momentum, Phys. Fluids 13, 3783 (2001).

[11] D. G. Dommermuth, J. W. Rottman, G. E. Innis, and E. A. Novikov, Numerical Simulation of the Wake of a Towed Sphere in a Weakly Stratified Fluid, J. Fluid Mech. 473, 83 (2002).

[12] J. M. Chomaz, P. Bonneton, A. Butet, and E. J. Hopfinger, Vertical Diffusion in the Far Wake of a Sphere Moving in a Stratified Fluid, Phys. Fluids A 5, 2799 (1993a).

[13] P. Meunier and G. R. Spedding, A Loss of Memory in Stratified Momentum Wakes, Phys. Fluids 16(2), 298 (2004).

[14] P. Meunier and G. R. Spedding, Stratified Propelled Wakes, J. Fluid Mech. 552, 229 (2006).

[15] J. R. Riley and S. M. deBruynKops, Dynamics of turbulence strongly influenced by buoyancy, Phys. Fluids 15(7), 2047 (2003).

[16] H. Tennekes and J. L. Lumley, A First Course in Turbulence (M.I.T. Press, Cambridge, Ma, USA, 1972).

[17] G. R. Spedding, Anisotropy in Turbulence Profiles of Stratified Wakes, Phys. Fluids 
13(8), 2361 (2001).

[18] Y. D. Afanasyev, Wakes behind towed and self-propelled bodies: Asymptotic theory, Phys. Fluids 16, 3235 (2004).

[19] P. M. Bevilaqua and P. S. Lykoudis, Turbulence Memory in Self-Preserving Wakes, J. Fluid Mech. 89, 589 (1978).

[20] J. R. Riley, R. W. Metcalfe, and M. A. Weissman, in Nonlinear Properties of Internal Waves, edited by N. Y. B. J. West (AIP (1981), pp. 79-112.

[21] M. J. Lighthill, Internal Waves and Related Initial-Value Problems, Dyn. Atmos. Ocean 23, 3 (1996).

[22] E. Itsweire, J. Koseff, D. Briggs, and J. H. Ferziger, Turbulence in Stratified Shear Flows: Implications for Interpreting Shear-Induced Mixing in the Ocean, J. Phys. Ocean. 23, 1508 (1993).

[23] W. D. Smyth and J. N. Moum, Anisotropy of turbulence in stably stratified mixing layers, Phys. Fluids 12, 1343 (2000).

[24] M. L. Waite and P. Bartello, Stratified turbulence dominated by vortical motion, J. Fluid Mech. 517, 281 (2004).

[25] D. K. Lilly, Stratified Turbulence and the mesoscale variability of the atmosphere, J. Atm. Sciences 40(3), 749 (1983).

[26] P. Billant and J.-M. Chomaz, Self-similarity of strongly stratified inviscid flows, Phys. Fluids 13, 1645 (2001).

[27] P. J. Diamessis, A. J. Domaradzki, and G. R. Spedding, Scaling laws of high Reynolds number turbulent stratified wakes in LES, subm. to Phys. Fluids (2006).

[28] O. Praud, A. M. Fincham, and J. Sommeria, Decaying grid turbulence in a strongly stratified fluid, J. Fluid Mech. 522, 1 (2005).

[29] O. Praud and A. M. Fincham, The structure and dynamics of dipolar vortices in a stratified fluid, J. Fluid Mech. 544, 1 (2005).

[30] C. H. Gibson, in Marine Turbulence, edited by J. C. T. Nihoul (Elsevier, 1980), pp. $221-258$. 
[31] S. Gallet, P. Meunier, and G. R. Spedding, Empirical scaling of antisymmetric stratified wakes, to appear in J. Fluids Struct. (2006).

[32] P. J. Diamessis, A. J. Domaradzki, and J. S. Hesthaven, A spectral multidomain penalty method model for the simulation of high Reynolds number localized incompressible stratified turbulence, J. Comp. Phys. 202, 298 (2005). 


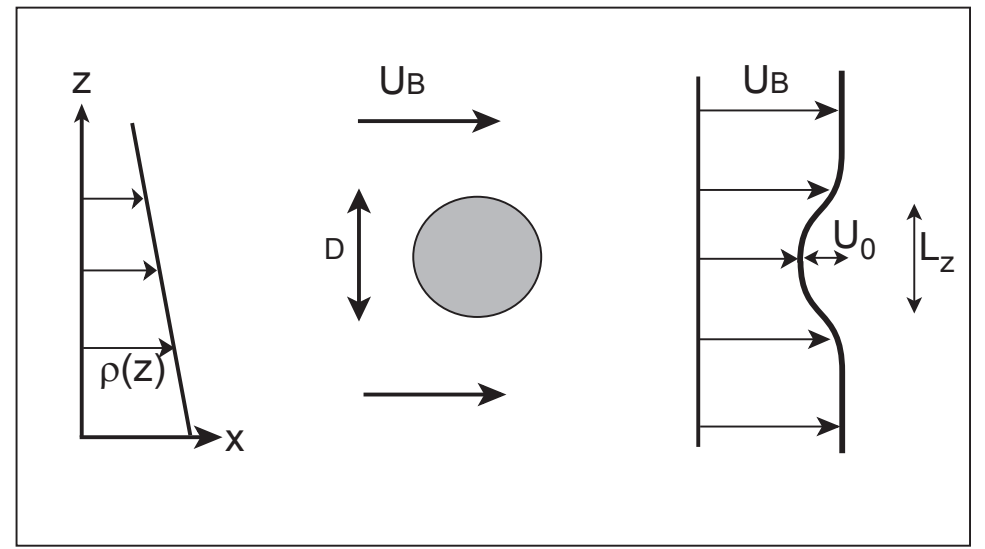

FIG. 1: Schematic of the wake created by a bluff body of diameter $D$ translated horizontally at $U_{B}$ in a linearly stratified fluid.

\section{FIGURES}




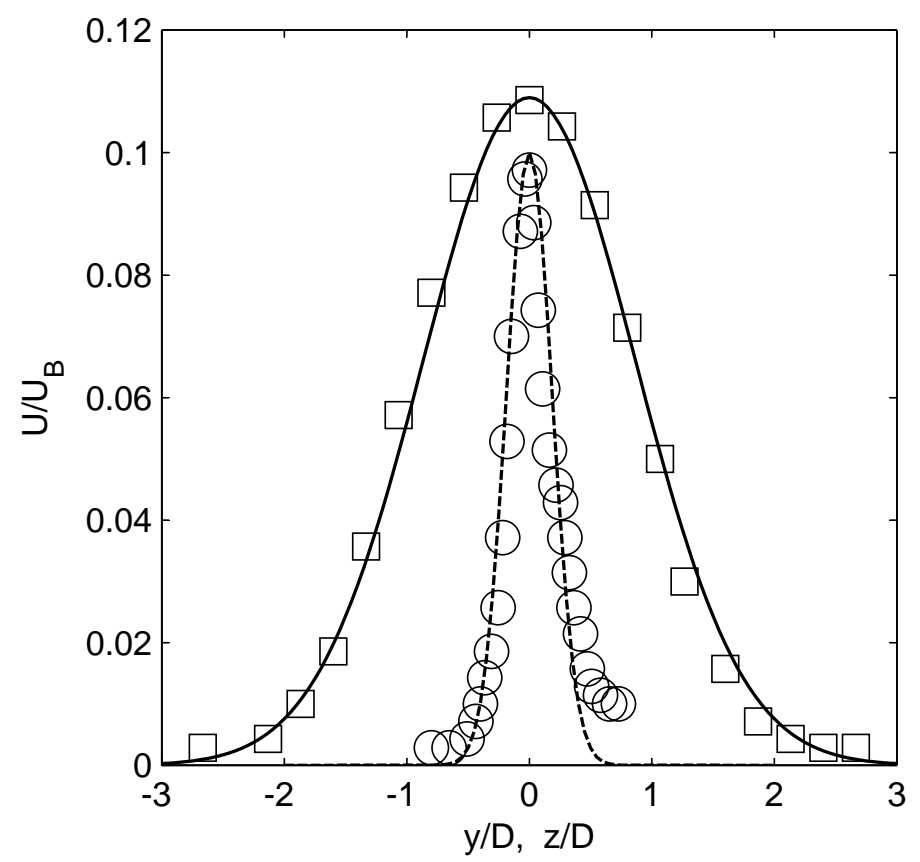

(a)

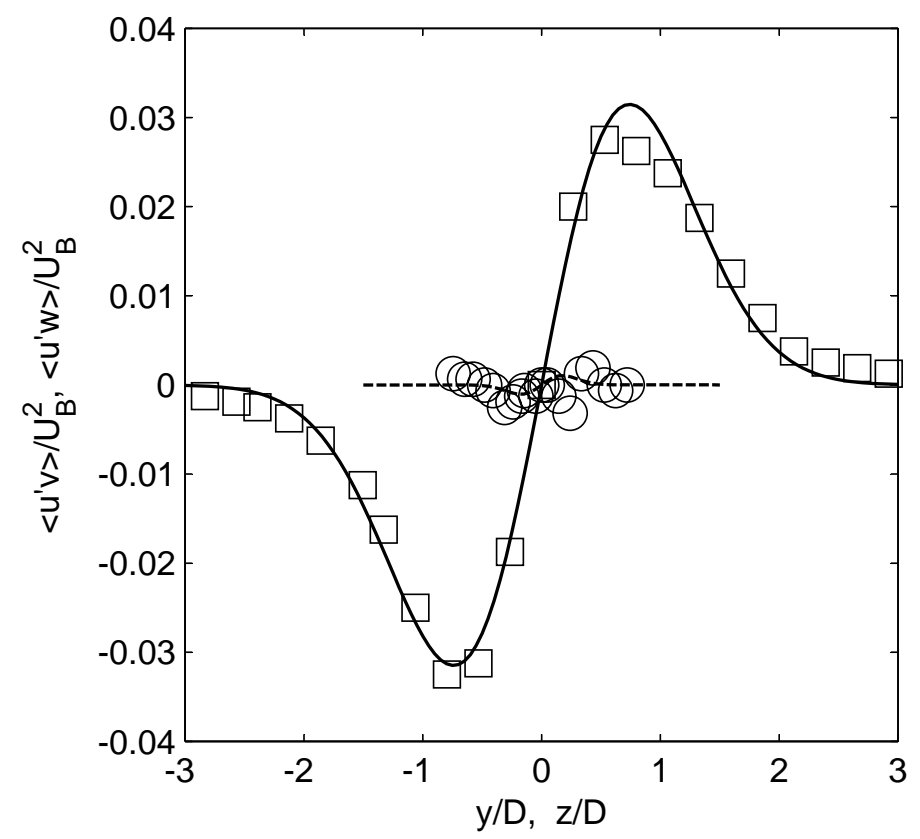

(b)

FIG. 2: Profile of (a) the mean velocity $U$ and (b) the Reynolds stresses $\left\langle u^{\prime} v^{\prime}\right\rangle$ and $<u^{\prime} w^{\prime}>$ for a sphere wake at $N t=20$ for $F=4$ and $R e=5 \times 10^{3}$. The data come from experimental results by [17] in the horizontal $(\square)$ and vertical centreplane (o). The solid lines show a Gaussian profile for the mean velocity and the derivative of a Gaussian profile for the Reynolds stresses. 


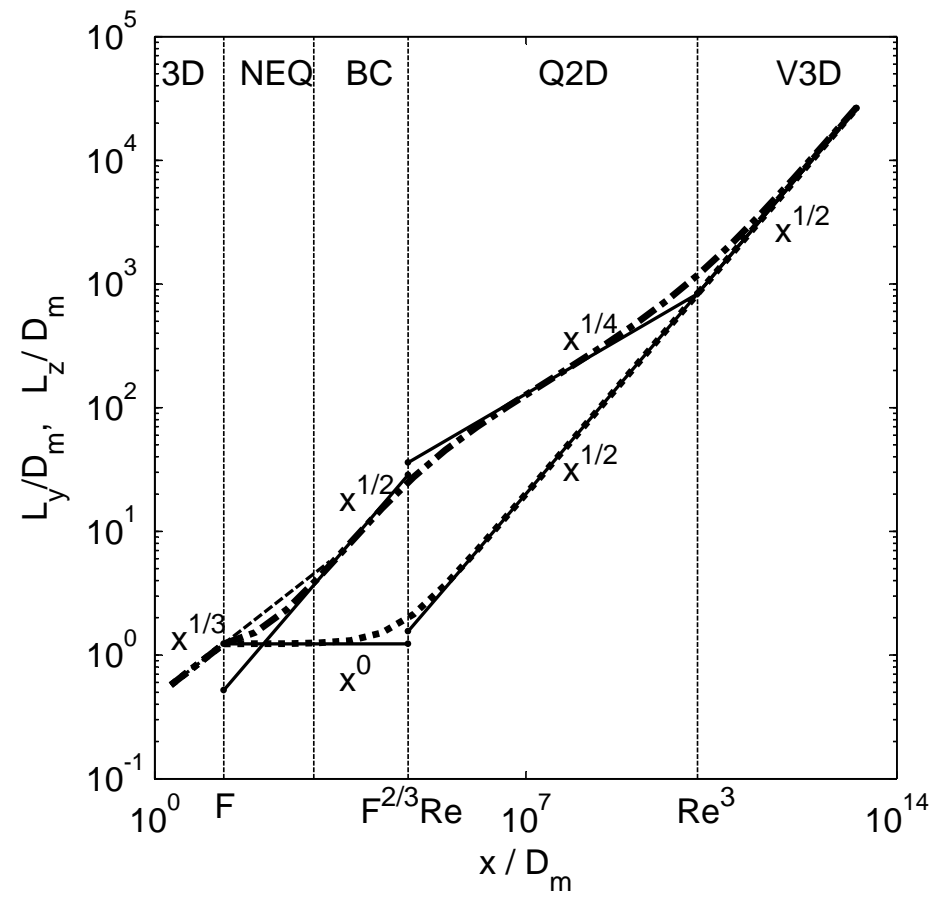

(a)

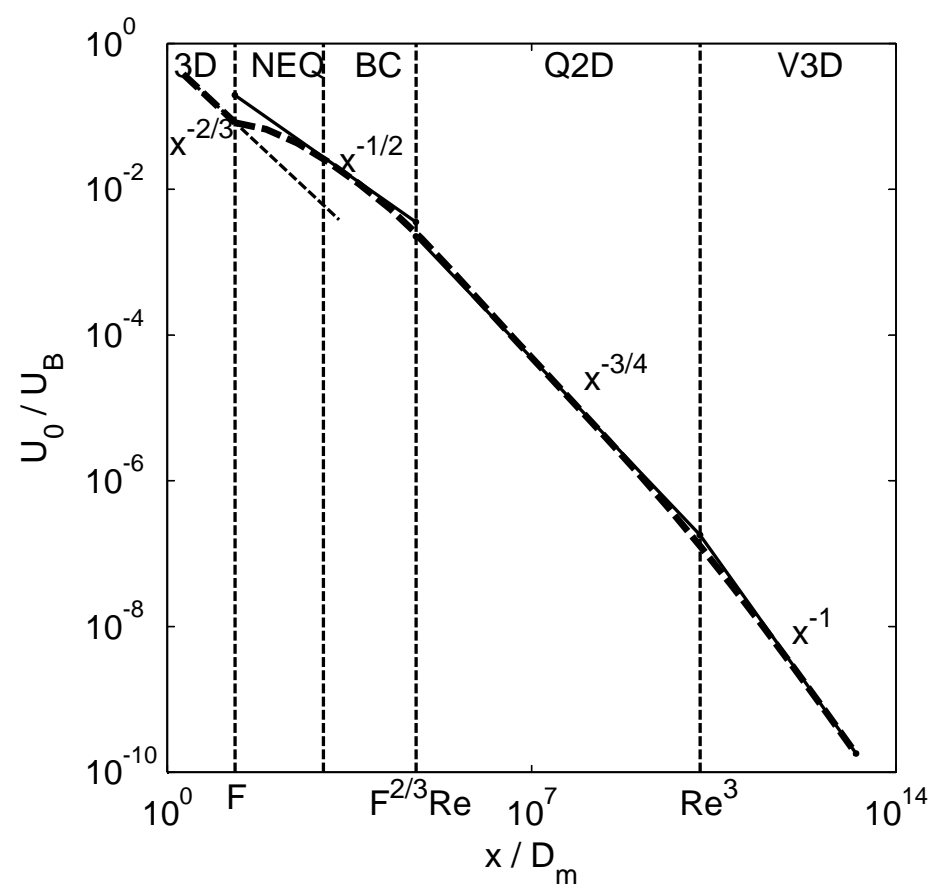

(b)

FIG. 3: Evolution of (a) the wake height $L_{z}$ (dotted line) and wake width $L_{y}$ (dash-dotted line), and of (b) the velocity defect $U_{0}$, given by the model: Eqs. (20), (22) and (7) respectively. The thin solid lines correspond to the asymptotic predictions for each stage. The thin dashed line corresponds to the standard theory for a homogeneous fluid $(13),(14) . F_{\mathrm{m}}=20$ and $R e_{\mathrm{m}}=50 \times 10^{3}$ 


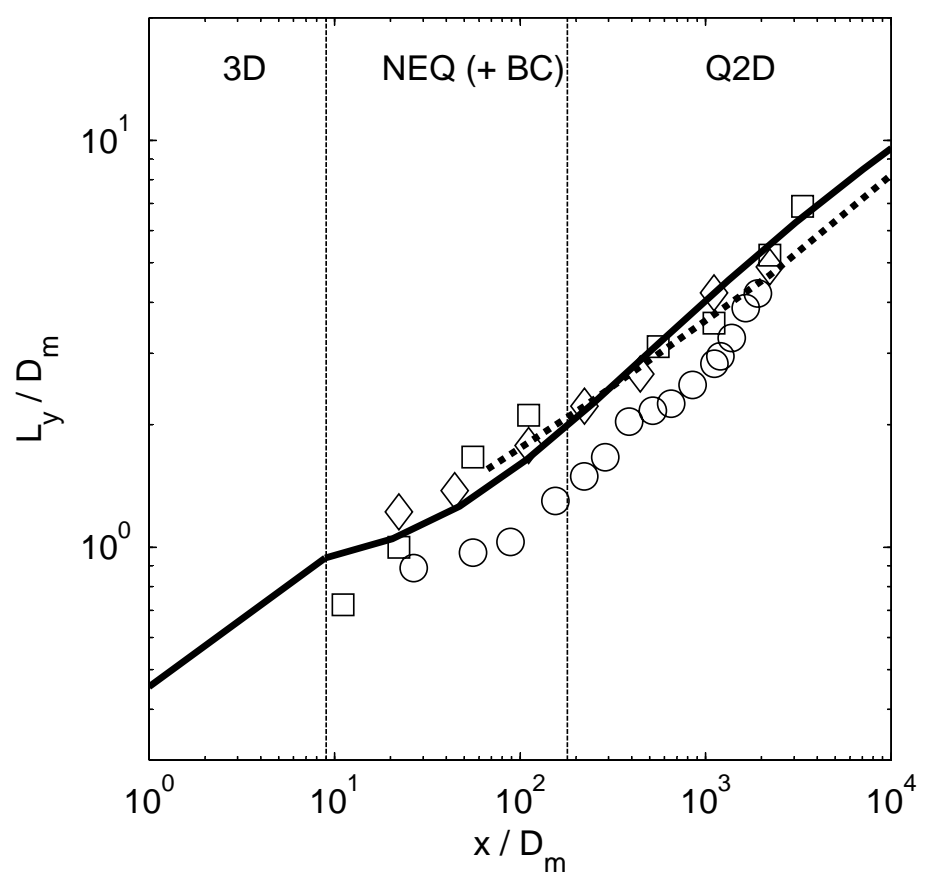

FIG. 4: Time evolution of the wake width $L_{y}$ for a sphere at $F_{m}=8.9$ and $R e_{m}=2.25 \times 10^{3}$. The model predictions (solid line) are compared with the experimental results from [4] (dotted line). Numerical simulations (open symbols) from [1] $(\diamond)$ for $F_{m}=8.9, R e_{m}=$ $4.5 \times 10^{3}$, from [10] ( $\square$ ) for $F_{m}=22.2, R e_{m}=4.5 \times 10^{3}$ and from [32] (o) for $F_{m}=8.9$, $R e_{m}=2.25 \times 10^{3}$ are also shown. The uncertainties in the experimental results [4] is of the order of $25 \%$. 


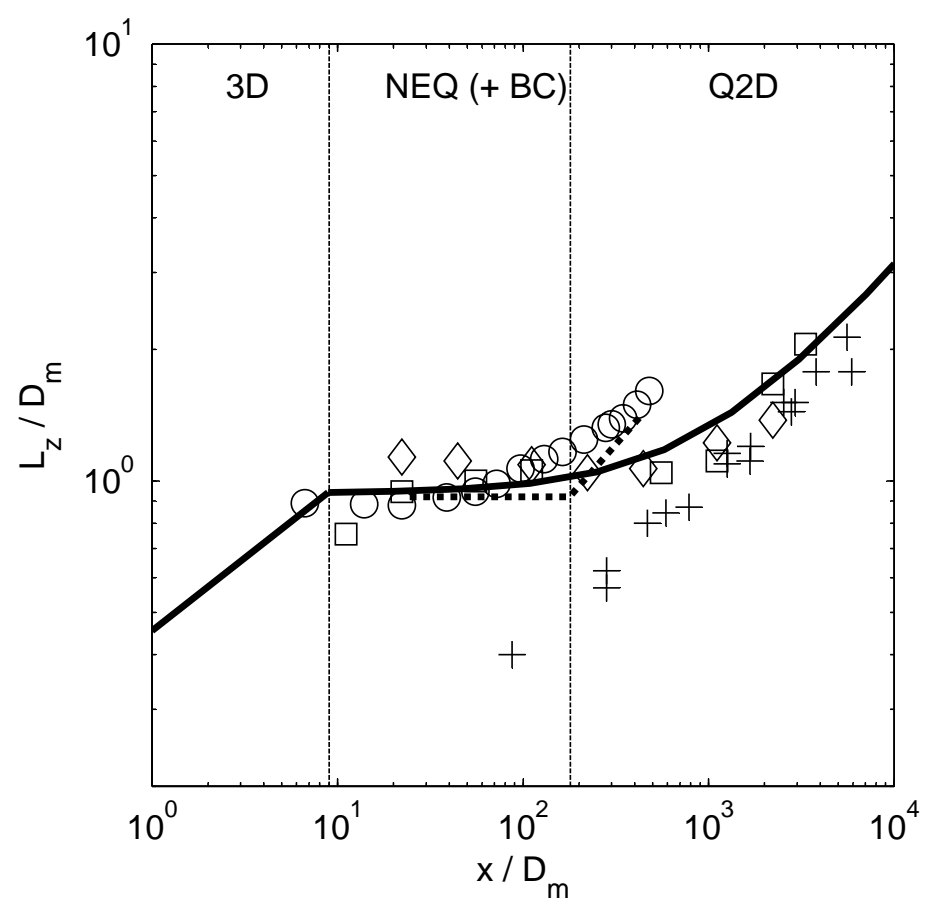

FIG. 5: Temporal evolution of the wake height $L_{z}$ for $F_{m}=8.9$ and $R e_{m}=2.25 \times 10^{3}$. Same caption as in Fig. 4. Experimental results from [12] for $F_{m} \approx 8.9$ and $R e_{m} \approx 2.25 \times 10^{3}$ are also shown by + symbols. The uncertainties in the experimental results [4] is of the order of $25 \%$. 


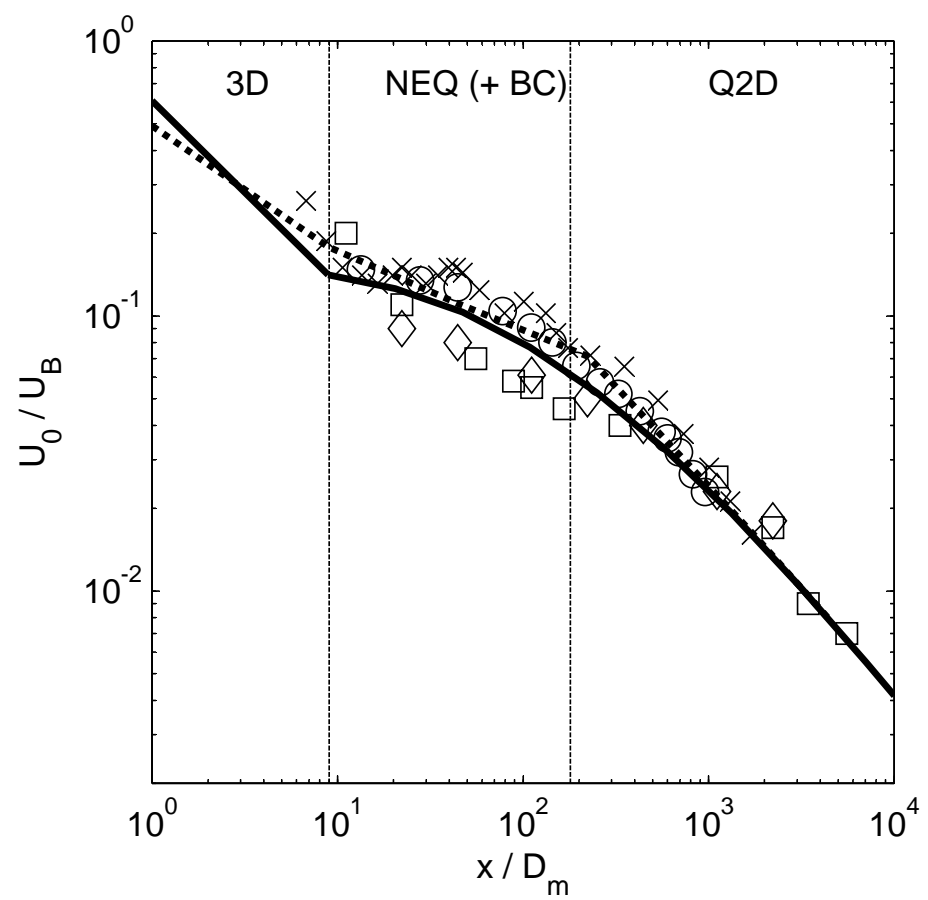

FIG. 6: Time evolution of the mean velocity defect $U_{0}$ for $F_{m}=8.9$ and $R e_{m}=2.25 \times 10^{3}$. Same caption as in Fig. 4. Experimental results from [8] for $F_{m}=13.3$ and $R e_{m} \approx 3 \times 10^{3}$ are also shown as $\times$ symbols. The uncertainties in the experimental results [4] is of the order of $40 \%$. 


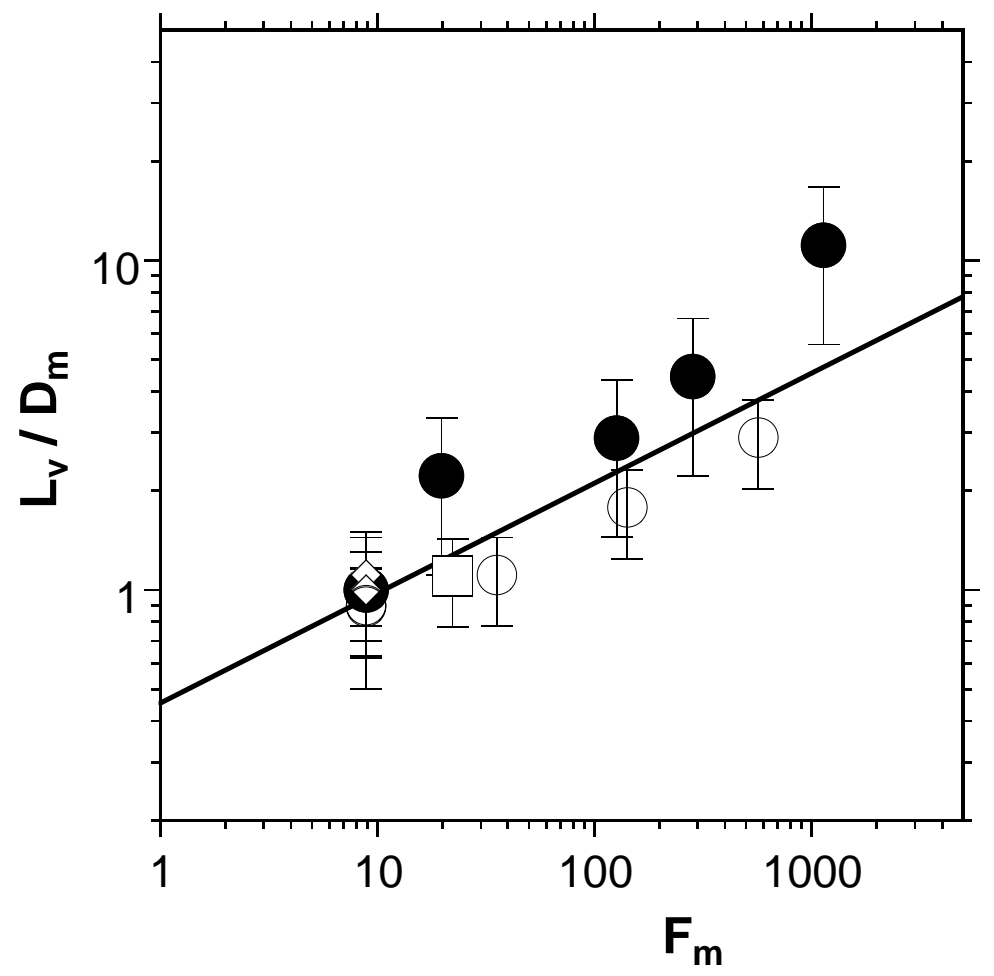

FIG. 7: Variation of the initial vertical wake height with Froude number. Experimental

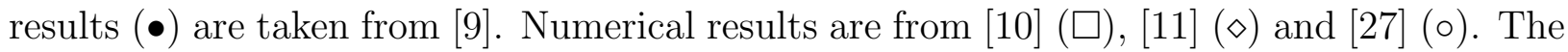
solid line corresponds to Eq. (15). 

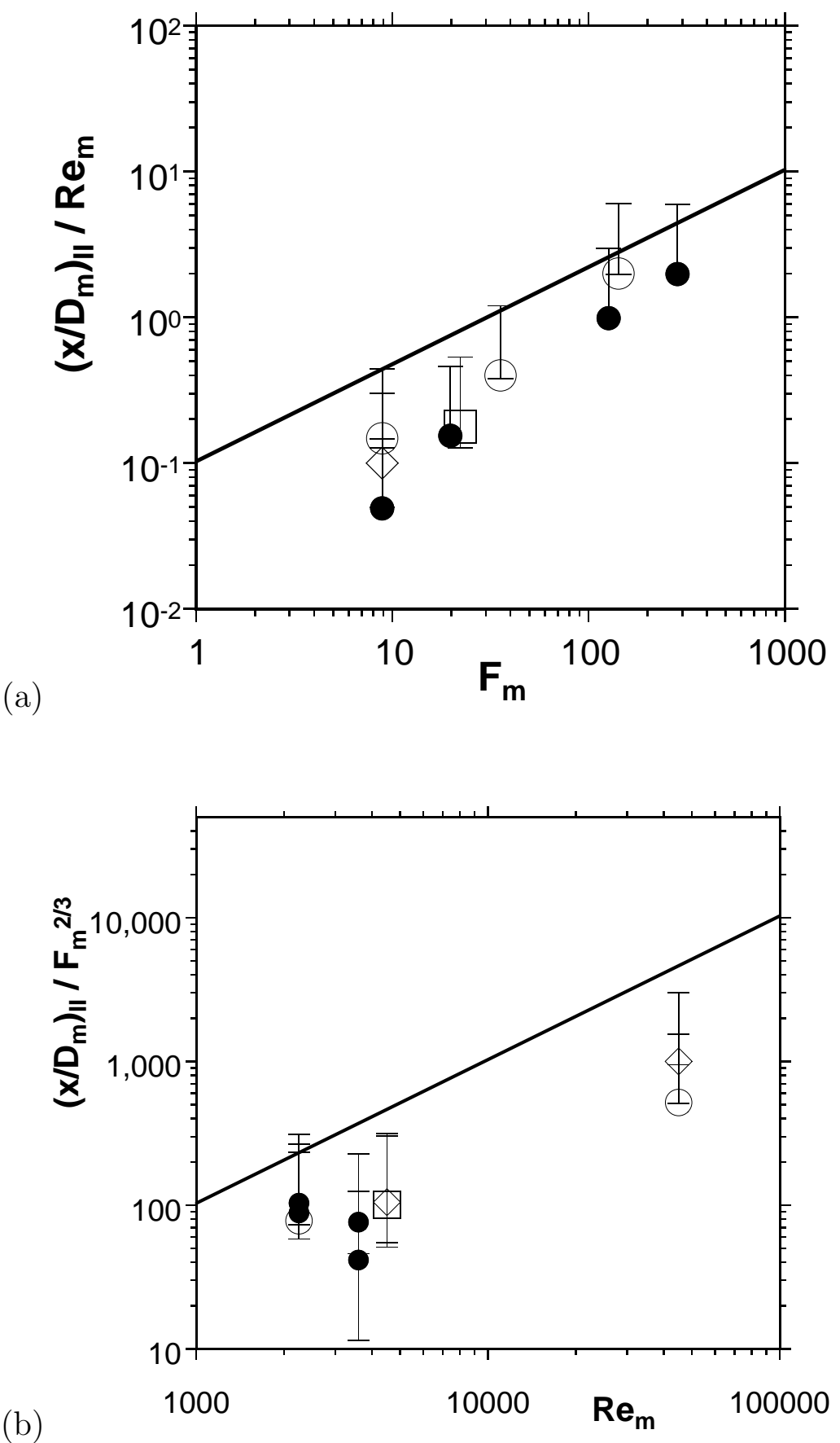

FIG. 8: Downstream distance $x_{I I}$ of the second transition, between a buoyancy-controlled (BC) regime and a quasi-2D regime (Q2D), as a function of (a) the Froude number and (b) the Reynolds number. Caption as in Fig. 7. The solid line corresponds to Eq. (21). 